\title{
Cisto periodontal lateral em maxila mimetizando cisto residual: Relato de caso incomum
}

\section{Lateral Periodontal Cyst in Maxilla Mimicking Residual Cyst: Unusual Case Report}

\author{
Damião Edgleys Porto \\ Especialista em Cirurgia e Traumatologia Buco-Maxilo-Facial pela \\ Universidade Estadual da Paraíba, UEPB, Campina Grande, PB.
}

Hellen Bandeira de Pontes Santos Mestranda do Programa de Pós-Graduação em Odontologia da Universidade Estadual da Paraíba, UEPB, Campina Grande, PB.

Livia Natália Sales Brito Mestranda do Programa de Pós-Graduação em Odontologia da Universidade Estadual da Paraíba, UEPB, Campina Grande, PB.

Gustavo PINA Godoy Professor Doutor, Departamento de Patologia, Universidade Federal de Pernambuco, UFPE, Recife, PE.

Pollianna Muniz Alves

Professora Doutora, Programa de Pós-Graduação em Odontologia, Universidade Estadual da Paraíba, UEPB, Campina Grande, PB.

Cassiano Francisco Weege Nonaka Professor Doutor, Programa de Pós-Graduação em Odontologia, Universidade Estadual da Paraíba, UEPB, Campina Grande, PB.

\section{RESUMO}

Introdução: O cisto periodontal lateral $(\mathrm{CPL})$ é um cisto odontogênico de desenvolvimento incomum. Essa lesão acomete predominantemente a mandíbula, e é comumente observada lateralmente à raiz de um dente vital. Em raros casos, essa lesão pode permanecer mesmo após a exodontia do dente associado e se assemelhar, radiograficamente, a um cisto residual. Objetivo: Relatar um caso incomum de CPL em maxila que mimetizava, radiograficamente, um cisto residual. Além disso, serão abordadas suas características clínico-patológicas, diagnóstico diferencial e terapêutica para essa lesão. Relato do caso: Paciente de 76 anos de idade apresentava uma lesão nodular na região palatina de rebordo alveolar, na área correspondente aos dentes 13 e 14. O exame radiográfico da região afetada demonstrou uma imagem radiolúcida unilocular e bem delimitada. Sob a hipótese diagnóstica de cisto residual, procedeu-se à enucleação da lesão. Após a análise histopatológica, os achados microscópicos foram compatíveis com CPL. Quatorze meses após a remoção cirúrgica da lesão, sinais clínicos ou radiográficos de recidiva não foram constatados. Conclusão: Em virtude da possibilidade do CPL permanecer mesmo após a exodontia do dente associado e se assemelhar, radiograficamente, a um cisto residual, o diagnóstico dessa lesão deve ser baseado nos achados histopatológicos e menor importância deve ser dada à sua localização adjacente ou lateral à raiz de um dente vital.

Palavras-chave: Cistos odontogênicos; cisto periodontal; cistos maxilomandibulares; diagnóstico diferencial; radiografia.

\section{ABSTRACT}

Introduction: The lateral periodontal cyst (LPC) is an uncommon developmental odontogenic cyst. This lesion affects mainly the mandible and is commonly seen laterally to the root of a vital tooth. In rare cases, this lesion may remain even after extraction of the associated tooth and resemble a residual cyst in radiographic exams. Objective: To report a case of LPC in maxilla that mimicked radiographically a residual cyst. In addition, it will also be discussed clinicopathologic features, differential diagnosis, and treatment for this lesion. Case report: A 76-year-old patient presented with a nodular lesion in the palate region of the alveolar ridge, in the area 
corresponding to teeth \#13 and \#14. Radiographic examination of the affected area showed a unilocular and well-delimited radiolucency. Under the diagnostic hypothesis of a residual cyst, enucleation of the lesion was performed. After histopathological analysis, microscopic features were compatible with LPC. Fourteen months after the surgical excision, clinical or radiographic signs of recurrence were not detected. Conclusion: Due to the possibility of LPC remain even after extraction of the associated tooth and resemble a residual cyst in radiographic exams, diagnosis of this lesion should be based on histopathological findings and less importance should be given to its location adjacent or lateral to the root of a vital tooth.

Keywords: Odontogenic cysts; periodontal cyst; jaw cysts; differential diagnosis; radiography.

\section{INTRODUÇÃO}

O cisto periodontal lateral (CPL) é um cisto odontogênico de desenvolvimento raro e representa aproximadamente $0,2 \%$ de todos os cistos que acometem os ossos gnáticos, ${ }^{1}$ bem como 0,6 a $2 \%$ de todos os cistos odontogênicos..$^{2-6}$ Fatores relacionados à sua etiopatogênese continuam pouco entendidos. ${ }^{7-8}$ Tem sido sugerido que essa lesão pode ser oriunda de remanescentes da lâmina dentária ou a partir da descamação de porções laterais do epitélio reduzido do órgão do esmalte em uma direção apical antes da erupção da coroa dentária na cavidade oral. $^{8}$

Essa lesão cística ocorre com maior frequência em indivíduos entre a quarta e a sétima décadas de vida, sendo discretamente mais frequente em homens. ${ }^{1,8-9}$ A maioria dos casos ocorre na mandíbula, principalmente entre as raízes de caninos e pré-molares. ${ }^{8-10}$ Radiograficamente, o CPL apresenta-se como uma área radiolúcida, com formato arredondado, geralmente localizado entre o ápice radicular e a margem cervical de dentes com vitalidade. ${ }^{8}$ Embora raro, há casos de CPLs que permaneceram mesmo após a exodontia dos dentes associados, mimetizando cistos residuais radiograficamente..$^{7,11-12}$

Mesmo nos casos mais típicos, caracterizados por uma lesão radiolúcida unilocular associada à superfície lateral de canino/pré-molares inferiores com vitalidade pulpar, os achados clínico-radiográficos do CPL não são suficientes para estabelecer o diagnóstico dessa lesão. ${ }^{10}$ Eventualmente, outras lesões odontogênicas podem ter apresentação clínico-radiográfica similar e, dessa forma, para o diagnóstico definitivo, faz- se necessária a análise histopatológica. ${ }^{7,8-10,13}$ Microscopicamente, o CPL apresenta uma cápsula fibrosa delgada e um revestimento composto por 1-3 camadas de epitélio pavimentoso ou cuboidal não ceratinizado.,9-10 Frequentemente, há a presença de placas ou espessamentos no revestimento epitelial, e acúmulos focais de células claras ricas em glicogênio podem ser observados tanto nas placas epiteliais, como nas camadas mais superficiais desse revestimento. ${ }^{7,9}$

A enucleação cirúrgica é o tratamento de escolha para o CPL e caso a lesão esteja associada a dentes vitais, maior cuidado no momento da cirurgia faz-se necessário. ${ }^{8-10,13}$ Na maioria dos casos, o prognóstico do CPL

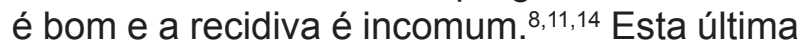
tem sido relacionada à variante botrioide do CPL, cuja recorrência é observada em aproximadamente $32,4 \%$ dos casos, ${ }^{15}$ ou pode constituir doença residual, em decorrência da remoção incompleta da lesão. ${ }^{14}$

Este trabalho objetivou relatar um caso incomum de $\mathrm{CPL}$ em maxila que mimetizava, radiograficamente, um cisto residual, abordando suas características clínico-patológicas, diagnóstico diferencial e terapêutica para essa lesão.

\section{Relato de CAso}

Paciente do gênero feminino, 76 anos de idade, leucoderma, procurou o ambulatório do Serviço de Cirurgia e Traumatologia Bucomaxilofacial do Hospital Regional Felipe Tiago Gomes - Picuí - Paraíba - Brasil, queixando-se de aumento de volume na região de palato. Durante a anamnese, foi relatado que a lesão estava presente há aproximadamente 
10 anos. A paciente apresentava um bom estado de saúde geral e negou história de tabagismo. Além disso, não apresentava linfonodos cervicais alterados à palpação.

Ao exame físico intraoral, verificou-se que a paciente era edêntula total e apresentava uma lesão nodular, exofítica, com coloração semelhante à da mucosa, localizada na região palatina de rebordo alveolar, na área correspondente aos dentes 13 e 14. A lesão, de consistência firme e crescimento lento, se apresentava bem delimitada e possuía aproximadamente $20 \mathrm{~mm}$ de diâmetro, clinicamente assemelhando-se a uma neoplasia de glândula salivar, neoplasia mesenquimal ou processo proliferativo não neoplásico (Figura 1). O exame radiográfico panorâmico revelou ausência de dentes inclusos ou restos radiculares, tanto em mandíbula quanto em maxila, bem como a presença de imagem radiolúcida unilocular em região de rebordo alveolar da maxila direita (Figura 2A). Por sua vez, o exame radiográfico periapical da região afetada revelou uma imagem radiolúcida unilocular, de formato circular e contornos regulares, delimitada por halo radiopaco e exibindo proximidade com o seio maxilar direito (Figura 2B).

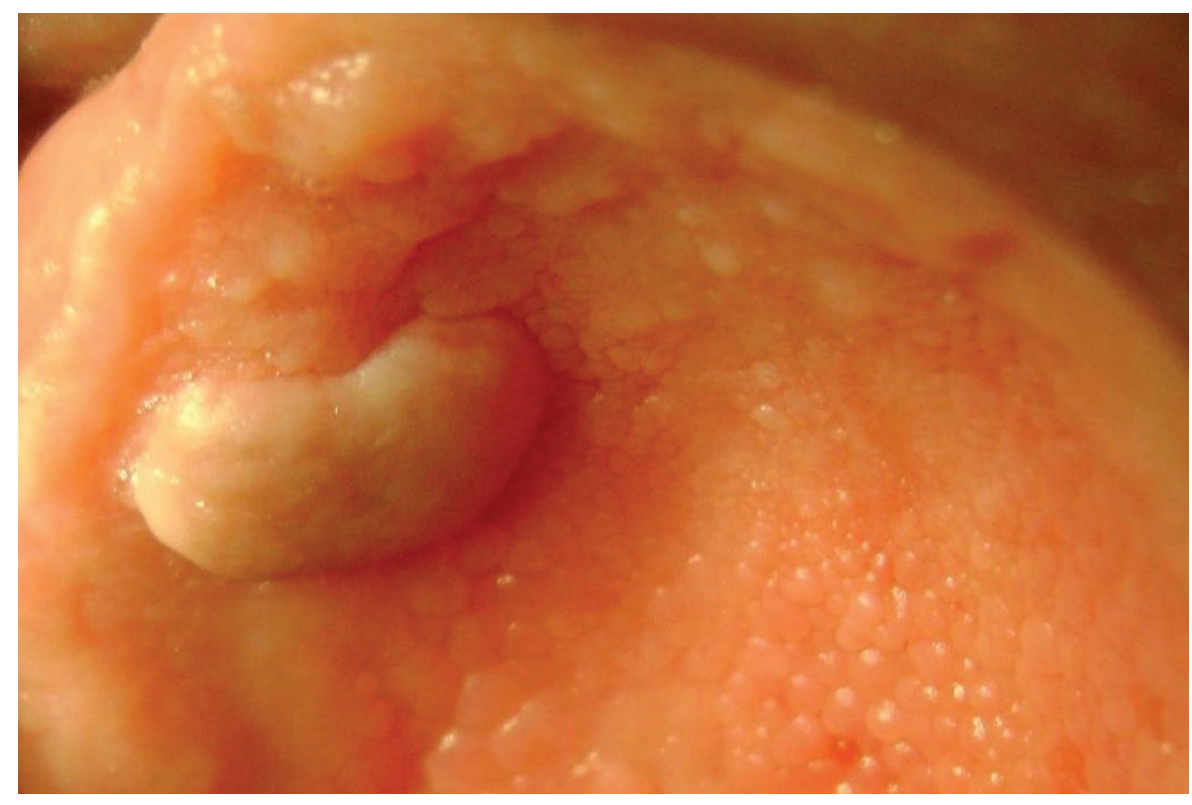

Figura 1 - Lesão nodular, com coloração semelhante à da mucosa, localizada na região palatina de rebordo alveolar, na área correspondente aos dentes 13 e 14.

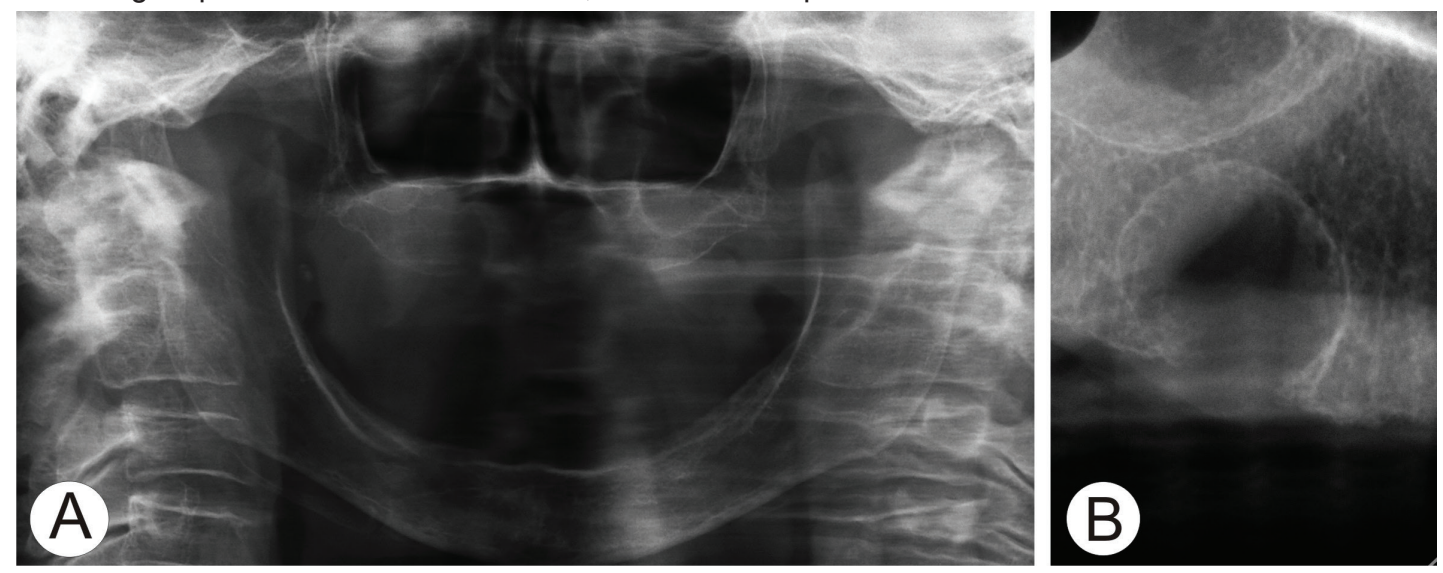

Figura 2 - A) Radiografia panorâmica mostrando ausência de dentes inclusos ou restos radiculares, bem como a presença de imagem radiolúcida unilocular em região de rebordo alveolar da maxila direita. B) Radiografia periapical evidenciando área radiolúcida, circunscrita por halo radiopaco em região anterior de maxila. 
Baseado nos achados clínicos e radiográficos foi estabelecida a hipótese diagnóstica de cisto residual. Dessa forma, procedeu-se à enucleação da lesão, sob anestesia geral e entubação nasotraqueal. Foi realizada uma incisão em forma de "U", que se estendeu do fundo de sulco vestibular correspondente à região do dente 15 ao fundo de sulco vestibular da região do dente 12 . Posteriormente, procedeu-se o descolamento do retalho mucoperiosteal, de modo a expor a região vestibular. Foram realizadas osteotomias com broca esférica, sob irrigação constante com solução de soro fisiológico a $0,9 \%$. Após a exposição, promoveu-se a remoção da lesão, bem como o debridamento e limpeza da área. Em sequência, o retalho foi suturado com pontos separados e foi instituído

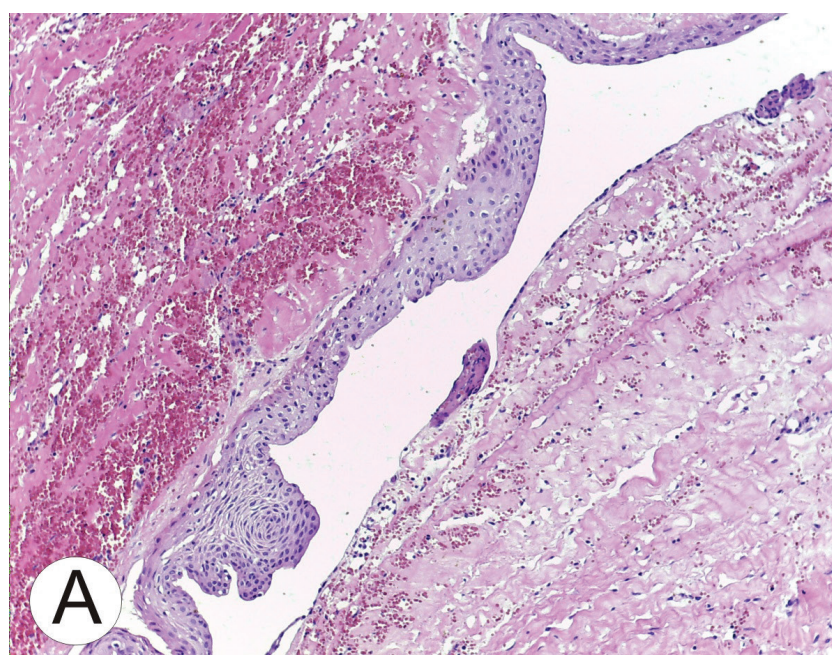

antibioticoterapia (Cefalexina, $500 \mathrm{mg}, 8 \mathrm{~h} / 8 \mathrm{~h}$, durante 15 dias), anti-inflamatório (Ibuprofeno, $600 \mathrm{mg}, 8 \mathrm{~h} / 8 \mathrm{~h}$, durante 5 dias), bem como bochechos com digluconato de clorexidina a 0,12\% (durante 10 dias).

A peça cirúrgica foi fixada em formol a $10 \%$ e encaminhada para exame histopatológico. Este revelou uma cavidade cística revestida por epitélio estratificado não ceratinizado, de poucas camadas, que exibia interface plana com a cápsula fibrosa circunvizinha e apresentava células superficiais com morfologia ora cuboidal ora pavimentosa (Figura 3A). Ao longo do revestimento epitelial, foram identificados espessamentos focais que se projetavam em direção ao lúmen cístico, bem como focos de células claras com citoplasma amplo (Figura 3B).

Figura 3 -A) Fotomicrografia revelando cavidade cística revestida por epitélio pavimentoso estratificado não ceratinizado, com espessamentos focais projetando-se para o lúmen cístico (coloração em H.E. e aumento de 100x). B) Focos de células claras com citoplasma amplo (coloração em H.E. e aumento de 400x).

Com base nos achados microscópicos, foi estabelecido o diagnóstico definitivo de CPL. Após 14 meses da remoção cirúrgica da lesão, foi verificado neoformação óssea local, e sinais clínicos ou radiográficos de recidiva não foram constatados (Figuras 4A e 4B). A paciente permanece sob proservação periódica.

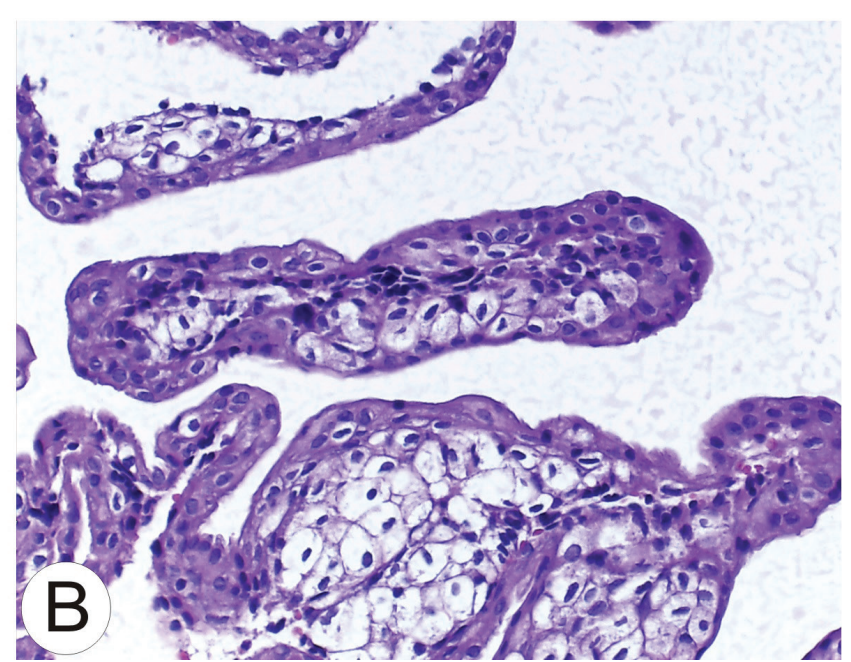



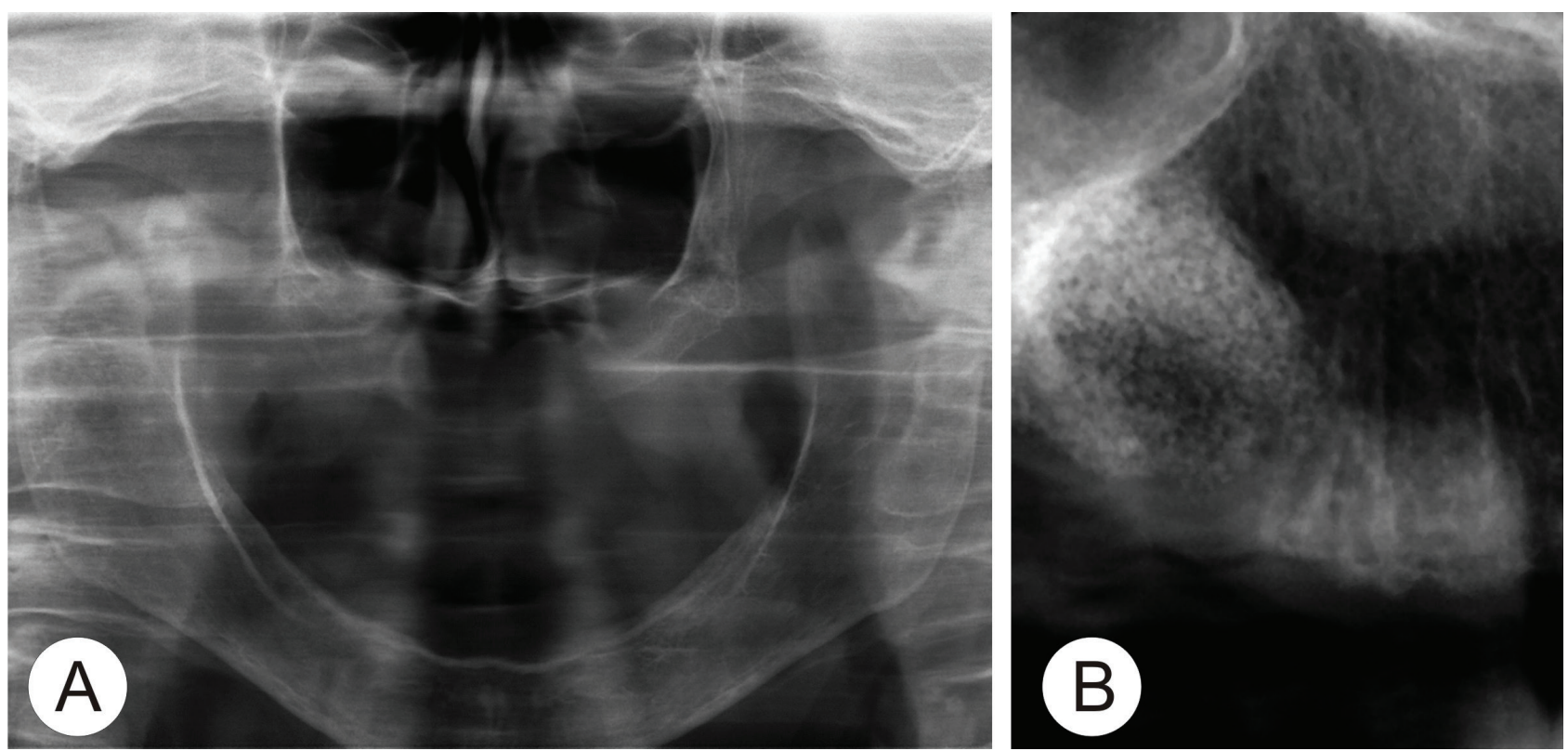

Figura 4 - A) Radiografia panorâmica de controle pós-operatório de 14 meses. B) Radiografia periapical evidenciando aumento da radiopacidade óssea local, compatível com neoformação óssea.

molares. ${ }^{9}$ Quando presente na maxila, essa lesão acomete principalmente a região anterior, similarmente ao presente caso. ${ }^{9,14}$

Essa lesão cística, assintomática e de crescimento lento, geralmente exibe pequenas dimensões no momento do diagnóstico, com diâmetros que variam de 3 até $10 \mathrm{~mm} \cdot{ }^{9-11} \mathrm{CPLs}$ de maiores dimensões têm sido relatados na maxila. ${ }^{14}$ Assim, o diâmetro do caso ora relatado $(20 \mathrm{~mm})$ pode estar relacionado à menor densidade óssea da maxila, que parece favorecer o maior crescimento dessa lesão, ou ao atraso no diagnóstico, uma vez que a paciente relatou apresentá-la há aproximadamente 10 anos.

Radiograficamente, a maioria dos casos de CPL caracteriza-se como uma área radiolúcida de forma circular ou ovoide, geralmente com margens escleróticas, localizada lateralmente à raiz de um dente vital. ${ }^{8,10}$ Embora menos usual, Mendes e van der Wall ${ }^{7}$ relataram dois casos de CPL em um local de extração prévia, mimetizando cistos residuais nos exames clínicos e radiográficos, similarmente ao presente caso. Diante desses casos não associados à superfície lateral de dentes vitais, Mendes e van der Wall ${ }^{7}$ destacam que é preferível definir essa lesão com base nos achados histopatológicos e dar, no contexto das lesões intraósseas do complexo maxilomandibular, menos importância à sua relação com os dentes. Por outro lado, devese ressaltar a importância da localização dessa lesão em relação aos tecidos moles e tecidos duros, pois o cisto gengival do adulto, contraparte dos tecidos moles do CPL, compartilha as mesmas características histopatológicas do CPL. ${ }^{10}$

Em virtude desses casos esporádicos de CPLs não associados à superfícies laterais radiculares de dentes vitais, além do fato dessas lesões realizarem diagnóstico diferencial com outras radiolucências nos exames radiográficos,,$^{7,8-10,12-13}$ ressalta-se a importância da análise histopatológica para o correto diagnóstico dessa lesão. A avaliação microscópica do presente caso revelou uma cápsula fibrosa e um revestimento de poucas camadas de epitélio estratificado não ceratinizado. Além disso, focos de células 
claras com citoplasma amplo e espessamentos focais projetavam-se em direção ao lúmen. Tais achados são similares ao que é comumente reportado na literatura para o CPL..$^{7,9-10}$

Devido à baixa tendência a recidivas do CPL, o presente caso foi tratado por meio de enucleação cirúrgica, procedimento esse

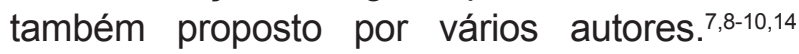
Ademais, quando a lesão está associada a dentes, cuidados no momento da cirurgia se fazem necessários, a fim de não proporcionar hipersensibilidade pulpare/ou perda devitalidade pulpar. $^{8,9-10,13} \mathrm{O}$ tratamento endodôntico pode ser necessário, caso o paciente apresente intensa hipersensibilidade pulpar após a cirurgia. ${ }^{13} \mathrm{~A}$ recorrência não é usual em CPLs ${ }^{9,11,14}$ e tem sido associada à doença residual ou à variante multicística dessa lesão, denominada cisto odontogênico botrioide. ${ }^{11,14-15}$

O caso apresentado evidencia a importância da remoção completa e curetagem de lesões císticas associadas a dentes, bem como o acompanhamento radiográfico após a cirurgia. Além disso, em virtude da possibilidade do CPL permanecer mesmo após a exodontia do dente associado e se assemelhar, radiograficamente, a um cisto residual, deve-se diagnosticar essa lesão com base nos achados histopatológicos e dar menos importância à sua localização adjacente ou lateral à raiz de um dente vital.

\section{REFERÊNCIAS}

1. Grossmann SM, Machado VC, Xavier GM, Moura MD, Gomez RS, Aguiar MC, et al. Demographic profile of odontogenic and selected nonodontogenic cysts in a Brazilian population. Oral Surg. Oral Med. Oral Pathol. Oral Radiol Endod., 2007; 104(6): e35-41.

2. de Souza LB, Gordón-Núñez MA, Nonaka CF, de Medeiros MC, Torres TF, Emiliano GB. Odontogenic cysts: demographic profile in a Brazilian population over a 38-year period. Med. Oral Patol. Oral Cir. Bucal, 2010; 15(4): e583-90.
3. Sharifian MJ, Khalili M. Odontogenic cysts: a retrospective study of 1227 cases in an Iranian population from 1987 to 2007; J. Oral Sci., 2011; 53(3): 361-7.

4. Selvamani M., Donoghue M., Basandi PS. Analysis of 153 cases of odontogenic cysts in a South Indian sample population: a retrospective study over a decade. Braz. Oral Res., 2012; 26(4): 330-4.

5. Johnson NR, Savage NW, Kazoullis S., Batstone MD. A prospective epidemiological study for odontogenic and non-odontogenic lesions of the maxilla and mandible in Queensland. Oral Surg. Oral Med. Oral Pathol. Oral Radiol. Oral Endod., 2013; 115(4): 515-22.

6. Dias D., Gazolla C., Matos B., Grossmann S., Oliveira L. Perfil epidemiológico de pacientes com diagnóstico de quisto odontogénico em uma universidade de odontologia. Rev. Port. Estomatol. Med. Dent. Cir. Maxillofac., 2014; 55(4): 238-42.

7. Mendes RA, Van der Waal I. An unusual clinicoradiographic presentation of a lateral periodontal cyst-report of two cases. Med. Oral Pathol. Oral Cir. Bucal, 2006; 11(2): E185-7.

8. Friedrich RE, Scheuer HA, Zustin J. Lateral periodontal cyst. In Vivo, 2014; 28(4): 595-8.

9. Andrade M., Silva AP, de Moraes Ramos-Perez FM, Silva-Sousa YT, da Cruz Perez DE. Lateral periodontal cyst: report of case and review of the literature. Oral Maxillofac. Surg., 2012; 16(1): 83-7.

10. de Carvalho LF, Lima CF, Cabral LA, Brandão AA, Almeida JD. Lateral periodontal cyst: a case report and literature review. J. Oral Maxillofac. Res., 2011; 1(4): e5.

11. Rasmusson LG, Magnusson BC, Borrman $\mathrm{H}$. The lateral periodontal cyst. A histopathological and radiographic study of 32 cases. Br. J. Oral Maxillofac Surg., 1991; 29(1): 54-7.

12. Siponen M., Neville BW, Damm DD, Allen CM. Multifocal lateral periodontal cysts: a report of 4 cases and review of the literature. Oral Surg. Oral Med. Oral Pathol. Oral Radiol. Oral Endod., 2011; 111(2): 225-33.

13. Dubey KN, Garg S., Atri R. Diagnosis and osseous healing of a lateral periodontal cyst mimicking a deep unusual interdental pocket in 
a young patient. Contemp. Clin. Dent., 2010; 1(1): 47-50.

14. Formoso Senande MF, Figueiredo R., Berini Aytés L., Gay Escoda C. Lateral periodontal cysts: a retrospective study of 11 cases. Med. Oral Patol. Oral Cir. Bucal, 2008; 13 (5): E313-7.

15. Méndez P., Junquera L., Gallego L., Baladrón J. Botryoid odontogenic cyst: clinical and pathological analysis in relation to recurrence. Med. Oral Patol. Oral Cir. Bucal, 2007; 12(8) E594-8.

Submetido em: 24-11-2015

Aceito em: 14-12-2015

\section{Autor correspondente:}

Cassiano Francisco Weege Nonaka 